\title{
From Powers to Exponential Function
}

\author{
Patricia Sureda ${ }^{1^{*}}$, Rita Otero ${ }^{1}$ \\ ${ }^{1}$ CONICET- NIECYT - UNICEN, ARGENTINA \\ *CORRESPONDENCE: $\square$ psureda@exa.unicen.edu.ar
}

\begin{abstract}
The construction of the exponential function of a real exponent from the definition of the powers of a real number, and its properties, requires the notion of successions, a decimal approximation of a rational number, convergence, and limit of a function. In Argentina, this construction would exceed what could be taught at the secondary level, but it provides advanced students of teachers in mathematics, and teachers in practice, the possibility of discussing what happens in the case of irrational exponents of the type $a^{\pi}$, or discuss the validity of the properties of this type of power.
\end{abstract}

Keywords: teacher training, empowerment, exponential function

\section{INTRODUCTION}

In the Argentine high school, the teachers do not teach the properties of powers together with potential functions, nor with exponential functions. On the contrary, professors define the potentiation in the set of natural numbers as $x^{a}=x . x . x \ldots x$ where $x$ multiplies himself sometimes, and then define the properties of natural exponent potentiation, which are easily demonstrable. But then, during the study of the other numerical sets, they do not take up again the definition of power of a real number, nor its properties. This generates a conceptual distance between the natural power definition of a natural number and the exponential function, defined for base and real exponent.

On the other hand, although defining the exponential function of the real exponent from the basic operation of the powers seems to be the "most natural" form, its construction quickly presents conceptual difficulties that can not be studied in secondary school. Among other reasons, because the procedure requires the use of the properties of the real set, and specific methods of analysis such as the notions of succession, limit and continuity.

However, discussing these types of concepts is useful for practicing teachers, or for the last few years of teacher training in mathematics. For most of the time the teaching of exponential functions axiomatically collaborates with the trivialization that is made of them. For example, because it is common to present the exponential function from its definition, accepting its existence and its properties, most of the time there is not even an intuitive idea of what happens in the case of irrational exponents of type $a^{\pi}$, nor are the validity of the properties of this type of power discussed. The proposal of Carbonero (2002) is more intuitive, especially for a secondary school teacher - although the construction of all the elements requires sixty pages-. Viewed from the perspective of completeness, it is the same construction mentioned above, but enriched with the use of Weierstrass theories and the elementary properties of convergence of sequences. Seen from the perspective of a high school teacher, it is the formalization of the intuitive construction that is usually taught to students.

In this work we build the exponential function from the potentiation properties. From the theory of limits only the Law of the Sandwich is used, which allows it to be built independently of the differential calculus. Thus, we build the exponential function from the potentiation properties in a more or less rigorous way. We

Article History: Received 19 February $2019 \bullet$ Revised 27 February $2019 \bullet$ Accepted 19 April 2019

(C) 2019 by the authors; licensee Modestum Ltd., UK. Open Access terms of the Creative Commons Attribution 4.0 International License (http://creativecommons.org/licenses/by/4.0/) apply. The license permits unrestricted use, distribution, and reproduction in any medium, on the condition that users give exact credit to the original author(s) and the source, provide a link to the Creative Commons license, and indicate if they made any changes. 
begin with the study of the exponential function from the definition of the function of powers $a^{x}$ (with natural $x$ ), and we extend it for any real exponent $x$. The idea of this demonstration is intuitive and is based on decimal approximations of real numbers, starting with the relatively simple tools of the theory of numerical successions.

\section{PROGRESSIVE EXTENSION OF $a^{x}$ FOR ANY EXPONENT $x$ REAL}

The construction requires first consider the exponent $\mathrm{x}$ as a natural number, then as an integer and later as a rational number. We will complete this construction by defining $a^{x}$ with irrational $x$, although this last step requires us to use the notions of succession, convergent sequence, limit, upper bound and decimal approximation of a number. In this work, we are only going to quote the definitions related to the natural powers, whole and rational, to devote ourselves then to the construction of the exponential function. We will not cite the properties and their demonstrations because they are accessible in any mathematical analysis manual.

\section{Natural Power}

We define the function that each $x$ assigns $x^{n}$ in the following way:

$$
\begin{gathered}
f: \mathbb{R} \rightarrow \mathbb{R} \\
x \rightarrow x^{n}
\end{gathered}
$$

We also define $x^{1}=x$; from which it follows that $x^{n+1}=x^{n} x^{1}=x^{n} x$; for all $x \in \mathbb{R}$ and $\forall n \in \mathbb{N}$.

\section{Integers Power}

For the case of power functions: $x \rightarrow x^{n}$ for $x$ real and $n$ natural integer we define the function nth power as the function that each $x \in R$ assigns $x^{\wedge} n$.

$$
\begin{gathered}
f: \mathbb{R} \rightarrow \mathbb{R} \\
x \rightarrow x^{n}
\end{gathered}
$$

In particular, for the negative integers $(-n)$ we define the following power function:

$$
f(x)=x^{-n}=\left(\frac{1}{x}\right)^{n}=\frac{1}{x^{n}} \forall n \in \mathbb{N}
$$

Thus, we have defined the power $x^{z}$ for $x \neq 0$, and any $z \in Z$. On the other hand, as $0 \in Z$, we define $x^{0}=$ 1 , for $n=0$. This can be easily deduced by doing $x^{0}=x^{n-n}=\frac{x^{n}}{x^{n}}=1$. There are also other demonstrations. For example, for the case in which the exponential and logarithmic functions are defined, the potential function for the case where $\mathrm{n}$ is real and $n=0$ is deduced from $x^{0}=e^{0 \ln (x)}=e^{0}=1$.

\section{Rational Power}

For the powers of real base and rational exponent we define for $x>0$ a positive real number, and $r=\frac{p}{q}$ a rational number, with $q>0$ and $p / q$ irreducible; the application that to each real number $x>0$ corresponds to $x^{r}$ is:

$$
\begin{gathered}
f: R \rightarrow R \\
x \rightarrow x^{r} \\
x \rightarrow x^{p / q}
\end{gathered}
$$

If $\mathrm{q}$ is odd then $x^{r}$ takes a single value (positive), if $\mathrm{q}$ is even then the root takes two real values of opposite signs. This implies taking for all $x$ the same positive determination $x^{r}(>0)$. Affirm that $x^{r}=y$ takes a single non-negative real number $y>0$, implies assuming that $x^{r}=y$ has a single non-negative real solution. In other words, if we take, for example, $r=\frac{1}{n}$ we expect $y=x^{\frac{1}{n}}$ to satisfy $y^{n}=x$. This solution is by definition the nth root of a non-negative real number, whose proof of existence is made in two parts. In the first part it is proved that the function $f(x)=x^{n}$ is strictly increasing in $[0, \infty)$. In the second part the completeness axiom is used to prove the existence of $a$, such that $a$ is the only real solution of $x^{n}$. 


\section{Exponential Function}

To define the symbol $a^{x}$ being a positive real and real $x$ we need to prove that there exists the limit of the sequence $\left\{r_{n}\right\}_{n \in \mathbb{N}}$, such that $r_{n}$ is the nth decimal approximation of $x$. That is, we want to try that:

$$
a^{x}=\gamma=\lim _{n \rightarrow \infty} a^{r_{n}}
$$

Knowing that $f(x)=a^{x}$ for $a>1$ and that $x \in Q$.

The proof is going to be done in three parts. In the first part, we will define the sequence, their limit and their convergence. In the second part we define the notion of decimal approximation, and we proof the existence of two decimal approximations for $x \in Q$, by constructing two sequences $\left\{r_{n}\right\}_{n \in \mathbb{N}}$ and $\left\{s_{n}\right\}_{n \in \mathbb{N}}$ such that both converge to $x$. The first sequence $\left\{r_{n}\right\}_{n \in \mathbb{N}}$ that converges to $x$ is increasing, while the other sequence $\left\{s_{n}\right\}_{n \in \mathbb{N}}$ converges to $x$ the definition of the following form $s_{n}=r_{n}+\frac{1}{10^{n}}$. In the part three, we use this construction to prove that $a^{x}=\gamma=\lim _{n \rightarrow \infty} a^{r_{n}}$ exists.

Part 1: Definition of convergent sequence and existence of the limit of a sequence.

When the elements $\left\{a_{n}\right\}_{n \in \mathbb{N}}$ approach a certain real number $l$, as $\mathrm{n}$ becomes large, the sequence $\left\{a_{n}\right\}_{n \in \mathbb{N}}$ is said to converge to $l$. To specify this better, take an interval $(l-\varepsilon, l+\varepsilon)$, with $\mathcal{E}>0$. If the $\left\{a_{n}\right\}_{n \in \mathbb{N}}$ is close to, you must have $\left\{a_{n}\right\}_{n \in \mathbb{N}} \in(l-\mathcal{E}, l+\mathcal{E})$, for $n$ large enough. This justifies the following definition.

Definition: It is said that the sequence $\left\{a_{n}\right\}_{n \in \mathbb{N}}$ converges to $l \in \mathbb{R}$ if for all $\mathcal{E}>0$ there exists $N \in \mathbb{N}$ such that $\left|a_{n}-l\right|<\mathcal{E}, \forall n \geq N$. In such a case it is said that the sequence $\left\{a_{n}\right\}_{n \in \mathbb{N}}$ is convergent, and that its limit is $l$. This is written as follows:

$$
\lim _{n \rightarrow \infty} a_{n}=l
$$

If the limit $l$ does not exist, it is said that $\left\{a_{n}\right\}_{n \in \mathbb{N}}$ diverges.

Next, slogans 1 and 2, and the law of the sandwich, also called the fitting theorem, are enunciated, because this theorem states that if two functions tend to the same limit in a point, any other function that can be bounded between the two previous ones will have the same limit in the point. All three are necessary to construct the exponential function through successions of decimal approximations, and they are accepted as true because their demonstration is easily accessible.

Lemma 1: If $\left\{a_{n}\right\}_{n \in \mathbb{N}}$ converges, then its limit is unique.

Lemma 2: Every convergent sequence is bounded.

Law of the Sandwich: Given two sequences $\left\{b_{n}\right\}_{n \in \mathbb{N}}$ and $\left\{c_{n}\right\}_{n \in \mathbb{N}}$, convergent to $l$. It is assumed that there exists $n_{0}$ such that $b_{n} \leq a_{n} \leq c_{n}, \forall n \geq n_{0}$; then $\left\{a_{n}\right\}_{n \in \mathbb{N}}$ also converges to $l$.

Part 2: Definition of Decimal Approximation of a real number $x$; and statement of the theorem that proves the existence of decimal approximations for $x \in Q$.

Decimal Approximation: In the following, $D$ will be called the set of digits, that is, the set of natural numbers between 0 and 9: $D=\{0,1, \ldots, 9\}$.

A positive real number $x$ admits a decimal approximation of the form:

$$
\begin{gathered}
x=a+\frac{c_{1}}{10^{1}}+\frac{c_{2}}{10^{2}}+\cdots \frac{c_{n}}{10^{n}} \\
x=a+\sum_{k=1}^{n} \frac{c_{k}}{10^{k}}
\end{gathered}
$$

Whit $a \in \mathbb{Z} ; c_{k} \in D$, y $n \in \mathbb{N}$. Such an expression is usually written $a, c_{1} c_{2} \ldots c_{n}$.

Theorem: Given a real number $x$, there is a single sequence of natural $c_{n} \in\{1,2, \ldots, 9\}$ tal que:

$$
\frac{c_{1}}{10}+\cdots+\frac{c_{n}}{10^{n}} \leq x<\frac{c_{1}}{10}+\cdots+\frac{c_{n}+1}{10^{n}}, \forall n \in \mathbb{N}
$$

In addition, the sequence $\left\{r_{n}\right\}_{n \in \mathbb{N}}$ defined above is increasing and converges to $x$, while the sequence $\left\{s_{n}\right\}_{n \in \mathbb{N}}$ defined by $s_{n}=r_{n}+\frac{1}{10^{n}}$ is decreasing and converges to $x$. The test is available in basic courses of mathematical analysis. 
In synthesis, if $\left\{c_{n}\right\}_{n \in \mathbb{N}}$ is the succession of the previous theorem, it is said that $x$ has decimal approximation $0, c_{1} c_{2} \ldots$ and it is written $x=0, c_{1} c_{2} \ldots$

In general, if $x>0$ and $c_{0}:=[x]$ is defined, we obtain $x-c_{0} \in[0,1)$ if the decimal approximation of $x-c_{0}$ is zero, $0, c_{1} c_{2} \ldots$ we write $x=c_{0}, c_{1} c_{2} \ldots$

Definition: A real sequence $\left\{x_{n}\right\}_{n \in \mathbb{N}}$ that satisfies the following property:

$$
(\forall n, m \in \mathbb{N})\left(n<m \rightarrow x_{n} \leq x_{m}\right)
$$

It is called crescent. It is clear that the succession of decimal approximations of every positive real number $x$ is increasing; but it is also bounded superiorly and converges to its lowest upper bound: $x$. This property of the successions of decimal approximations is generalizable: every real succession that is increasing and bounded superiorly converges to its lowest upper bound. The proof of this result is analogous to that of the theorem according to which a positive real number $x$ the limit of the succession of its decimal approximations.

Note: in the case of decimal approximations of real numbers, the limit of the succession coincides with its minimum upper bound (or its maximum lower bound).

Part 3: In this step we are dedicated to building the exponential function. In principle we define $f(x)=a^{x}$ for $a>1$ and $x \in \mathbb{Q}$. For this we remember that there exist sequences $\left\{r_{n}\right\}_{n \in \mathbb{N}}$ and $\left\{S_{n}\right\}_{n \in \mathbb{N}}$, the first crescent and the second decreasing such that:

$$
r_{n} \leq x<s_{n}=r_{n}+\frac{1}{10^{n}} \forall n \in \mathbb{N}
$$

Since $a>1$ we have that $f(r)=a^{r}$ is strictly increasing in $\mathbb{Q}$. Then we know that:

$$
r_{1} \leq r_{n} \leq r_{n+1} \leq x \leq s_{n+1} \leq s_{n} \leq s_{1}, \forall n \in \mathbb{N}
$$

we have to:

$$
a^{r_{1}}<a^{r_{n}}<a^{r_{n+1}}<a^{s_{n+1}}<a^{s_{n}}<a^{s_{1}}, \forall n \in \mathbb{N}
$$

With this we show that the sequence $\left\{a^{r_{n}}\right\}_{n \in \mathbb{N}}$ is increasing and bounded superiorly by $a^{r_{1}}$, while the sequence $\left\{a^{s_{n}}\right\}_{n \in \mathbb{N}}$ is decreasing and bounded below by $a^{r_{1}}$. By the Weierstrass theorem, which says that every monotonous and bounded sequence is convergent, we deduce that both sequences are convergent.

This allows us to define the following inheritance limits:

This allows us to define the following inheritance limits:

$$
\gamma=\lim _{n \rightarrow \infty} a^{r_{n}} \text { and } \mu=\lim _{n \rightarrow \infty} a^{s_{n}}
$$

On the other hand, knowing that the sequence $\left\{a^{1 / n}\right\}_{n \in \mathbb{N}}$ converges to one, we can deduce that as $10^{n}>n$ and then $\frac{1}{10^{n}}<\frac{1}{n}$, from which we deduce that $1<a^{1 / 10^{n}}<a^{1 / n}$.

Then, since $\left\{a^{1 / n}\right\}_{n \in \mathbb{N}}$ converges to one, then $\left\{a^{1 / 10^{n}}\right\}_{n \in \mathbb{N}}$ converges to one, we have to:

$$
\mu=\lim _{n \rightarrow \infty} a^{s_{n}}=\lim _{n \rightarrow \infty} a^{r_{n}+1 / 10^{n}}=\lim _{n \rightarrow \infty} a^{r_{n}} a^{1 / 10^{n}}=\gamma
$$

Now, since $\gamma$ is the only real number that is both greater than each $a^{r_{n}}$ and smaller than each $a^{s_{n}}$, if we want $\mathrm{f}$ to continue to be increasing, the only possible definition for $a^{x}$ is:

$$
a^{x}=\gamma=\lim _{n \rightarrow \infty} a^{r_{n}}
$$

This definition of the exponential function through the use of sequences greatly simplifies the work when demonstrating the properties of the exponential function for real exponents. However, the main tool to do it is the following motto.

Lemma: If $\left\{\alpha_{n}\right\}_{n \in \mathbb{N}}$ is a sequence of rationals that converges to $\mathrm{x}$, then the sequence $\left\{a^{\alpha_{n}}\right\}_{n \in \mathbb{N}}$ converges to $a^{x}$.

The demonstration of this motto requires the use of the Bernoulli inequality that is available in courses of mathematical analysis. Let's now try the following properties:

- For $a>1$ the function is strictly increasing in $\mathbb{R}$.

In fact, let $x$ and $y \in \mathbb{R}$ be such that $x<y$. Let $\left\{s_{n}\right\}_{n \in \mathbb{N}}$ and $\left\{t_{n}\right\}_{n \in \mathbb{N}}$ be sequences of rationals such that the first grows to $x$, and the second decreases to $y$. 
Let $\mathrm{p}$ and $q \in \mathbb{Q}$ such that $x<q<p<y$ then:

$$
a^{s_{n}}<a^{p}<a^{q}<a^{t_{n}}, \forall n \in \mathbb{N}
$$

Then,

$$
a^{x}=\lim _{n \rightarrow \infty} a^{s_{n}} \leq a^{p}<a^{q} \leq \lim _{n \rightarrow \infty} a^{t_{n}}=a^{y}
$$

- For $x$ and $y \in \mathbb{R}$ we have $a^{x+y}=a^{x} a^{y}$.

Taking $\left\{s_{n}\right\}_{n \in \mathbb{N}}$ and $\left\{t_{n}\right\}_{n \in \mathbb{N}}$ sequences of rationals such that: $\left\{s_{n}\right\}_{n \in \mathbb{N}}$ converges to $x$ and $\left\{t_{n}\right\}_{n \in \mathbb{N}}$ converges to $\mathrm{y}$; then $\left\{\mathrm{s}_{\mathrm{n}}+\mathrm{t}_{\mathrm{n}}\right\}_{\mathrm{n} \in \mathbb{N}}$ converges to $\mathrm{x}+\mathrm{y}$.

And for the previous lemma: $a^{x+y}=\lim _{n \rightarrow \infty} a^{s_{n}+t_{n}}=\lim _{n \rightarrow \infty} a^{s_{n}} a^{t_{n}}=a^{x} a^{y}$.

- For $x$ and $y \in \mathbb{R}$ we have $\left(a^{x}\right)^{y}=a^{x y}$.

Here you have to be a little more careful. First we assume that $x$ and $y$ are positive. Then we take three successions of positive rationals: $\left\{r_{n}\right\}_{n \in \mathbb{N}},\left\{s_{n}\right\}_{n \in \mathbb{N}}$ and $\left\{t_{n}\right\}_{n \in \mathbb{N}}$ such that $\left\{r_{n}\right\}_{n \in \mathbb{N}}$ grows to $x ;\left\{s_{n}\right\}_{n \in \mathbb{N}}$ decreases to $x$; and $\left\{t_{n}\right\}_{n \in \mathbb{N}}$ converges to $y$.

Then as $a^{r_{n}}<a^{x}$ then by the previous lemma we have to:

$$
a^{r_{n} t_{n}}=\left(a^{r_{n}}\right)^{t_{n}}<\left(a^{x}\right)^{t_{n}}
$$

and also by the previous lemma:

$$
a^{x y}=\lim _{n \rightarrow \infty} a^{r_{n} t_{n}} \leq \lim _{n \rightarrow \infty}\left(a^{x}\right)^{t_{n}}=\left(a^{x}\right)^{y}
$$

In a similar way

$$
a^{x y}=\lim _{n \rightarrow \infty} a^{s_{n} t_{n}} \leq \lim _{n \rightarrow \infty}\left(a^{x}\right)^{t_{n}}=\left(a^{x}\right)^{y}
$$

If we hit the two inequalities we obtain the equality sought. Then if $x<0$ or $y<0$ we have to proceed in a similar way.

The properties shown above are still valid for $0<a<1$, except that now the function $f(x)=a^{x}$ would be strictly decreasing.

\section{Characteristics of the exponential function: Continuity}

Let $a>1$, and the function $f(x)=a^{x}$ have just been defined. Recalling the inequality: $1<a^{\frac{1}{n}}<1+\frac{1}{n}$, for $a>1$ and $n \in N$.

Given $\mathcal{E}>0$, let $n \in \mathbb{N}$ such that $\frac{a}{n}<\mathcal{E}$. Then for $-\frac{1}{n}<x<\frac{1}{n}$ we have:

$$
a^{x}<a^{\frac{1}{n}}<1+\frac{a}{n}<1+\varepsilon
$$

And also:

$$
a^{x}>a^{-\frac{1}{n}}>\frac{1}{1+\varepsilon}>1-\varepsilon
$$

This proves that taking $\delta=\frac{1}{n}$ we have:

$$
|x|<\delta \Rightarrow\left|a^{x}-1\right|<\mathcal{E}
$$

This means that

$$
\lim _{x \rightarrow 0} a^{x}=1
$$

In other words, the exponential function is continuous at $x=0$. In general, making the change $t=x-x_{0}$ we have:

$$
\lim _{x \rightarrow x_{0}} a^{x}=\lim _{x \rightarrow x_{0}} a^{x_{0}} a^{x-x_{0}}=a^{x_{0}} \lim _{t \rightarrow 0} a^{t}=a^{x_{0}}
$$

and then the exponential function of base a is continuous throughout $\mathbb{R}$.

\section{Characteristics of the exponential function: Surjection}

Once we test continuity, we can use the intermediate values theorem to conclude that the range of the exponential function is all $\mathbb{R}^{+}$. 
If we consider a $y>0$ we observe that:

$$
a^{n}=(1+a-1)^{n}>n(a-1)
$$

For the Archimedean property there exists $n_{1} \in \mathbb{N}$ such that $n_{1}(a-1)>y$, where:

$$
a^{n_{1}}>n_{1}(a-1)>y
$$

In the same way there exists $n_{0} \in \mathbb{N}$ such that $n_{0}(a-1)>\frac{1}{y}$, where an $a n_{0}>\frac{1}{y}$. Consequently we have:

Consequently we have:

$$
a^{-n_{0}}<y<a^{n_{1}}
$$

By the theorem of the intermediate values, there exists $x \in\left(-n_{0}, n_{1}\right)$, such that $a^{x}=y$. With this we prove that the following function is surjective:

$$
f: \mathbb{R} \rightarrow(0, \infty), \quad f(x)=a^{x}
$$

As we already knew that it was injective (because it is strictly increasing), we conclude that it is in fact bijective.

Note: For $0<a<1$ we have $a^{x}=\frac{1}{b^{x}}$, with $b=\frac{1}{a}>1$, and it is not difficult to convince yourself that it is still bijective and continuous. From now on, when we talk about the exponential function, we will refer to the function defined by [1], with $a>0$ and $a \neq 1$.

In summary, everything said can be written as follows:

Theorem: The exponential function $f: \mathbb{R} \rightarrow(0, \infty), f(x)=a^{x}$ is bijective and continuous. Furthermore, it is strictly increasing if $a>1$, and strictly decreasing is $0<a<1$.

\section{Final Words}

In Argentina, the construction of the exponential function with real exponent from the potentiation that we have presented in this work, is not usually studied in the regular courses of analysis. However, its study in the mathematics education for teachers, allows to discuss the validity of the properties of this type of power, as well as to analyze issues, which from the functional point of view are accepted without questioning or simply ignored.

\section{ACKNOWLEDGEMENTS}

We grateful Dr. Ana Paula Madrid (Universidad Nacional del Centro de la Provincia de Buenos Aires) for the mathematical revision of the manuscript.

\section{Disclosure statement}

No potential conflict of interest was reported by the authors.

\section{Notes on contributors}

Patricia Sureda - Consejo Nacional de Investigaciones Científicas y Técnicas (CONICET)- Núcleo de Investigación en Educación en Ciencia y Tecnología (NIECYT) - Universidad Nacional del Centro de la Provincia de Buenos Aires (UNICEN), Argentina.

María Rita Otero - Consejo Nacional de Investigaciones Científicas y Técnicas (CONICET)- Núcleo de Investigación en Educación en Ciencia y Tecnología (NIECYT) - Universidad Nacional del Centro de la Provincia de Buenos Aires (UNICEN), Argentina. 


\section{REFERENCES}

Carbonero, S. (2002). Una Construcción Elemental de las Funciones Exponencial y Logarítmica. Matemática, Educación e Internet. 3(1), 1-56. Retrieved from http://revistas.tec.ac.cr/index.php/matematica/article/ view/2278

Friedelmeyer, J. P. (2005). Comment introduire les fonctions logarithmes et exponentielles au lycée? APMEP. 460, 645-664. Retrieved from https://www.apmep.fr/Comment-introduire-les-fonctions

Robledo Potes, J. (2014). Matemática fundamental para matemáticos. Cali (Colombia): Universidad del Valle.

http://www.iejme.com 